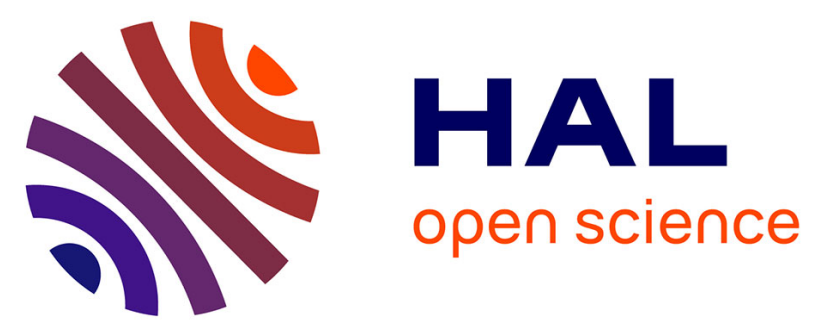

\title{
The sodium exosphere of Mercury: Comparison between observations during Mercury's transit and model results
} Alessandro Mura, Peter Wurz, Herbert I.M. Lichtenegger, Helmold Schleicher,

Helmut Lammer, Dominique C. Delcourt, Anna Milillo, Stefano Orsini, Stefano Massetti, Maxim L. Khodachenko

\section{To cite this version:}

Alessandro Mura, Peter Wurz, Herbert I.M. Lichtenegger, Helmold Schleicher, Helmut Lammer, et al.. The sodium exosphere of Mercury: Comparison between observations during Mercury's transit and model results. Icarus, 2009, 200 (1), pp.1. 10.1016/j.icarus.2008.11.014 . hal-00510969

\section{HAL Id: hal-00510969 \\ https://hal.science/hal-00510969}

Submitted on 23 Aug 2010

HAL is a multi-disciplinary open access archive for the deposit and dissemination of scientific research documents, whether they are published or not. The documents may come from teaching and research institutions in France or abroad, or from public or private research centers.
L'archive ouverte pluridisciplinaire HAL, est destinée au dépôt et à la diffusion de documents scientifiques de niveau recherche, publiés ou non, émanant des établissements d'enseignement et de recherche français ou étrangers, des laboratoires publics ou privés. 


\section{Accepted Manuscript}

The sodium exosphere of Mercury: Comparison between observations during Mercury's transit and model results

Alessandro Mura, Peter Wurz, Herbert I.M. Lichtenegger, Helmold Schleicher, Helmut Lammer, Dominique Delcourt, Anna Milillo, Stefano Orsini, Stefano Massetti, Maxim L. Khodachenko

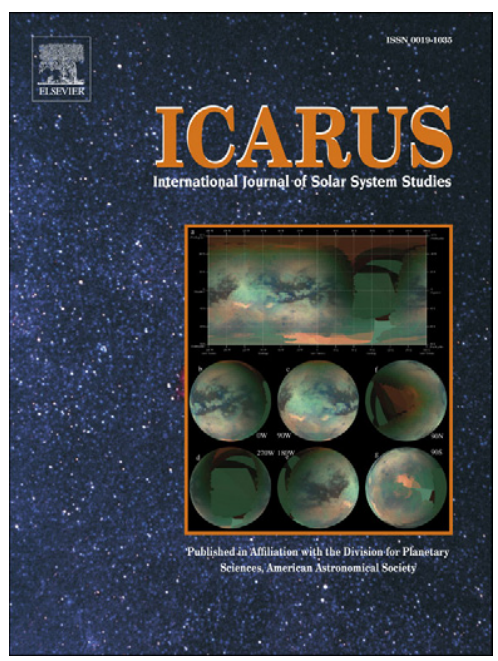

PII: $\quad$ S0019-1035(08)00410-7

DOI: $\quad$ 10.1016/j.icarus.2008.11.014

Reference: YICAR 8820

To appear in: Icarus

Received date: 29 April 2008

Revised date: 23 October 2008

Accepted date: 10 November 2008

Please cite this article as: A. Mura, P. Wurz, H.I.M. Lichtenegger, H. Schleicher, H. Lammer, D. Delcourt, A. Milillo, S. Orsini, S. Massetti, M.L. Khodachenko, The sodium exosphere of Mercury: Comparison between observations during Mercury's transit and model results, Icarus (2008), doi: 10.1016/j.icarus.2008.11.014

This is a PDF file of an unedited manuscript that has been accepted for publication. As a service to our customers we are providing this early version of the manuscript. The manuscript will undergo copyediting, typesetting, and review of the resulting proof before it is published in its final form. Please note that during the production process errors may be discovered which could affect the content, and all legal disclaimers that apply to the journal pertain. 
The sodium exosphere of Mercury: Comparison between observations during Mercury's transit and model results

Alessandro Mura ${ }^{\mathrm{a}}$, Peter Wurz ${ }^{\mathrm{b}}$, Herbert I.M. Lichtenegger ${ }^{\mathrm{c}}$, Helmold Schleicher ${ }^{\mathrm{d}}$, Helmut Lammer ${ }^{\mathrm{c}}$, Dominique Delcourt ${ }^{\mathrm{e}}$, Anna Milillo ${ }^{\mathrm{a}}$, Stefano Orsini ${ }^{\mathrm{a}}$, Stefano Massetti $^{\mathrm{a}}$, Maxim L. Khodachenko ${ }^{\mathrm{c}}$

${ }^{\text {a }}$ Istituto di Fisica dello Spazio Interplanetario-CNR, Rome, Italy

${ }^{\mathrm{b}}$ Physics Institute, University of Bern, Bern, Switzerland

${ }^{\mathrm{c}}$ Space Research Institute, Austrian Academy of Sciences, Graz, Austria

${ }^{\mathrm{d}}$ Kiepenheuer-Institut fuer Sonnenphysik, Freiburg, Germany

${ }^{\text {e }}$ CETP-CNRS, Saint Maurice des Fossés, France 
Proposed Running Head: The sodium exosphere of Mercury

Editorial correspondence to:

Dr. Alessandro Mura

Institute of Interplanetary Space Physics

INAF-IFSI Rome

Email: alessandro.mura@ifsi-roma.inaf.it 
Key Words: Mercury transit, sodium exosphere, surface release processes, chemical sputtering 


\section{ABSTRACT-}

4 In this study we compare the sodium exosphere observations made by Schleicher et 5 al.(2004) with the result of a detailed numerical simulation. The observations, made 6 during the transit of Mercury across the solar disk on 7 May 2003, show a maximum of

7 sodium emission near the polar regions, with north prevalence, and the presence of a 8 dawn-dusk asymmetry. We interpret this distribution as the resulting effect of two 9 combined processes: the solar wind proton precipitation causing chemical alteration of 10 the surface, freeing the sodium atoms from their bounds in the crystalline structure on the 11 surface, and the subsequent photon-stimulated and thermal desorption of the sodium 12 atoms. While we find that the velocity distribution of photon desorbed sodium can 13 explain the observed exosphere population, thermal desorption seems to play a minor role 14 only causing a smearing at the locations where $\mathrm{Na}$ atoms are released on the dayside. The 15 observed and simulated distributions agree very well with this hypothesis and indicate 16 that the combination of the proposed processes is able to explain the observed features. 


\section{Introduction}

In 1974, the UVE experiment on board Mariner 10 (Broadfoot et al., 1976) observed a tiny exosphere around Mercury, made of hydrogen, helium, and, probably, oxygen. Starting from 1985 (Potter and Morgan, 1985), many ground-based observations have identified the presence of sodium in Mercury's exosphere. Most of these observations indicate either north-south or dawn-dusk asymmetries (see Killen et al., 2007, for a recent review on observations). Several processes, such as ion sputtering (IS), thermal desorption (TD), photon-stimulated desorption (PSD), and micro-meteoroid vaporization, have been proposed for being responsible for the formation of the sodium exosphere (e.g., McGrath et al., 1986; Hunten et al., 1988; Potter and Morgan, 1997; Madey et al., 1998; Yakshinskiy and Madey, 1999; Killen et al., 1999, 2001, 2004, 2007; Leblanc and Johnson, 2003; Wurz and Lammer, 2003). In fact, Leblanc and Johnson (2003) concluded in their study that the sodium surface density distribution becomes significantly nonuniform from the day to the night side, and from low to high latitudes as well as from the morning to the afternoon due to the rapid depletion of $\mathrm{Na}$ atoms in the surfaces of grains mainly driven by thermal depletion. Killen et al. (2004) suggested that ion precipitation increases the diffusion from the interior.

Recently, Schleicher et al. (2004) published sodium tangential column densities, derived from observation of Mercury transit of May 2003. These column densities show a strong dawn-dusk asymmetry, an enhancement near the poles, and a moderate northsouth asymmetry. Also, the reported scale heights are several 100's of km, which is difficult to reconcile with thermal desorption using realistic temperatures. The aim of the present study is to investigate the possibility that these features may be the result of two combined processes:

1) space weathering of the surface by precipitating solar wind protons, which cause chemical liberation of sodium atoms at the surface, and

2) photon-stimulated desorption of the liberated sodium from the surface into the exosphere.

For this purpose we have developed a numerical model that is able to reproduce the proposed processes and which allows the comparison of the simulated data with the 
47 observations. Already in earlier observations $\mathrm{Na}$ and $\mathrm{K}$ were sometimes found in

48 localized enhancements at elevated latitudes, which was associated with solar wind entry 49 through the magnetospheric cusps. Also dawn-dusk asymmetries were observed earlier,

50 with pronounced $\mathrm{Na}$ and $\mathrm{K}$ dawn enhancements. A possible explanation for these features 51 was by recycling of $\mathrm{Na}$ and $\mathrm{K}$, which become ionized in the exosphere, enter the 52 magnetospheric system, and with some probability are implanted on the surface again 53 (Sprague, 1992; Hunten and Sprague, 1997). Diffusion will bring these atoms back to the 54 surface where they will thermally desorb. Diffusion of $\mathrm{Na}$ and $\mathrm{K}$ from locations in the 55 regolith to the surface has been studied in detail (e.g., Sprague 1992; Killen et al., 2004).

56 Since diffusion and desorption are both thermally driven processes, they will not occur on 57 the night side and implanted $\mathrm{Na}$ and $\mathrm{K}$ will accumulate there until Sun rises. 58 Accumulation of $\mathrm{Na}$ and $\mathrm{K}$ on the night side explains in a natural way the dawn-dusk 59 asymmetry and the enhancements at high latitudes, the latter because of the regions of ion 60 precipitation on Mercury's surface.

61 In Section 2 we give a brief outline of the observations; in Section 3 the numerical 62 model used to simulate the data is described. A discussion of the results is given in 63 Section 4 , followed by a summary and conclusion in Section 5.

\section{Observations}

66 On May 7, 2003, a transit of Mercury across the solar disk occurred. On the occasion of this optimal observation configuration, Schleicher et al. (2004) obtained a series of 2D spectra of the solar sodium resonance line at $589.0 \mathrm{~nm}$ (D2), with Mercury being positioned near the field-of-view during the $\sim 2$ hours of observation (UT 8:27 to 10:25). At each location $h$ above the limb and at azimuth $\varphi$, the column density along the line of

71 sight $n(h, \varphi)$ has been derived from the observed equivalent widths $W_{\lambda}(h, \varphi)$ from the 72 curve-of-growth relation for an absorption tube. For the Doppler-width of the absorption 73 profile the value measured from the observed averaged profile of the excess absorption 74 was used. The equations are given in the appendix section. The derived tangential column 75 densities are displayed in figure 1. 
These observations show a maximum $\mathrm{Na}$ density at the planetary limb, exponentially decreasing with altitude. The density around the limb, however, is not uniform: two maxima can be found near the polar regions, with a prevalence of the northern one. There, the Na column density along the line of sight (in the following referred as "tangential column density") is up to $8 \times 10^{10} \mathrm{~cm}^{2}$, and the estimated volume density at the surface is $2.6 \times 10^{3} \mathrm{~cm}^{3}$ (Schleicher et al., 2004); the presence of sodium is noticeable up to more than $700 \mathrm{~km}$ above the surface. Between the two peaks, along the dawn limb, a density excess can be seen. If we integrate the tangential column density over the entire field of view, we obtain a total $\mathrm{Na}$ content in the exosphere of $4 \times 10^{27}$ atoms. From the Doppler width of the line profile of the excess absorption, Schleicher et al. (2004) estimated that the parallel component of the velocity distribution was $1.6 \mathrm{~km} \mathrm{~s}^{-1}$, which may corresponds to a very high temperature $(\sim 3500 \mathrm{~K})$ if we assume a Maxwellian distribution of the particles.

\section{[Figure 1]}

\section{Model Description}

The general concept, underlying our simulations is that the surface abundance of sodium is depleted by thermal and photon-stimulated desorption, and refueled by the solar wind precipitation. In our study, we assume that for each solar wind proton precipitating onto the surface, with some probability a sodium atom will eventually be available at the surface, as the result of chemical alteration of the mineral grain (described below). Hence, we first evaluated the solar wind proton flux onto Mercury's surface corresponding to the solar activity conditions during the transit observations. Then we simulated the sodium surface composition, which was finally used as an input for our 3D exosphere model.

At the time of the considered observations of Mercury exosphere, the planet was 0.45 AU away from the Sun, at an anomaly angle of about $150^{\circ}$. The real physical conditions in the solar wind (in particular speed, density, IMF, etc.) and the solar irradiative 
105 background near Mercury during the transit are of critical importance for interpretation of

106 its exosphere observations and verification of the applied models. According to the

107 GOES satellite synoptic data, the Sun was relatively quiet during the whole transit of

108 Mercury on May 7, 2003, with just 3 X-ray events of C-class registered, of which only

109 one in the northern hemisphere (N15E88, C2.2 from 10:17 till 10:27) took place during

110 the performed observations, near the end of the transit. From these data it is justified that

111 we use as an input the average quiet Sun radiation flux in our model.

112 Because of the absence of in-situ measurements of the solar wind plasma parameters

113 near Mercury at the time of the transit, we used for their estimation the solar wind data

114 measured by the ACE satellite which is located downstream of Mercury at the Lagrange

115 point L1 at $1.5 \times 10^{6} \mathrm{~km}$ from the Earth. The fact that Mercury's transit was observed from

116 the Earth, and the ACE spacecraft is also located close to the Sun-Earth line, it makes it

117 possible to assume that the solar wind flow measured by ACE within the next 1 to 2 days

118 after the Mercury transit belongs to the same stream of the solar wind that passed near 119 Mercury.

120 Figure 2 shows the ACE data, measured components of magnetic field, solar wind 121 proton speed and density, as well as the distance from the spacecraft to Earth, during the 122 Mercury transit on May 7, 2003 (day of year 127) and two days after the transit 123 (http://www.srl.caltech.edu/ ACE/ASC/level2/index.html). As it can be seen, a relatively 124 fast interplanetary CME passed ACE with a velocity $\sim 890 \mathrm{~km} \mathrm{~s}^{-1}$ ) on May 9, 2003.

[Figure 2]

However, taking into account the speed of this CME and mutual location of Mercury 129 and the ACE spacecraft, one can calculate that the CME passed Mercury after the transit. 130 Similar treatment of other parts of the solar wind speed curve measured by ACE makes it 131 possible to conclude that the solar wind portion with the speed $\sim 780 \mathrm{~km} \mathrm{~s}^{-1}$, registered by 132 ACE on May 8, 2003, was very likely passing Mercury on May 7, 2003 during the transit 133 observations. Taking into account the decrease of the solar wind speed with distance from 
134 the Sun, we extrapolate ACE values down to the Sun and estimate the solar wind speed 135 near Mercury during the transit on May 7, 2003 as $\sim 800 \mathrm{~km} \mathrm{~s}^{-1}$. Using ACE magnetic

136 field measurements (see Figure 2) and taking into account the decrease of magnetic field 137 with distance $\left(\sim 1 / r^{2}\right)$, as well as solar wind motion, the components of the IMF at

138 Mercury during the transit on May 7, 2003 can be estimated as $(-20,10,-10) n T$.

139 The proton precipitation flux, which is needed for the exosphere modeling, was 140 obtained by using a single- particle model (Delcourt et al., 2003, Mura et al., 2005). The 141 original magnetic field model (Luhmann and Friesen 1979, Delcourt et al., 2003) was

142 adapted to the specific conditions at Mercury by adding the IMF field. The resulting 143 proton flux onto the surface has the general shape similar to that presented in Mura et al. 144 (2005). The total flux in the northern hemisphere is about $4 \times 10^{25} \mathrm{~s}^{-1}$, while the negative 145 IMF $x$-component introduces a flux enhancement in the southern hemisphere similar to 146 the results in Sarantos et al. (2001).

\subsection{Chemical alteration of the surface composition}

The impact of energetic ions (solar wind or magnetospheric ions) on the surface 150 affects the topmost layer of the regolith. The induced processes are ion implantation, 151 sputtering of surface material, and chemical alteration, where the latter allows the 152 breaking of existing and creation of new chemical compounds. Production of sodium and 153 water by proton sputtering of sodium-bearing silicates was considered by the following 154 mechanism (Potter, 1995)

$$
2 \mathrm{H}+\mathrm{Na}_{2} \mathrm{SiO}_{3} \rightarrow 2 \mathrm{Na}+\mathrm{SiO}_{2}+\mathrm{H}_{2} \mathrm{O}:
$$

The free energy of this reaction is $-4.7 \mathrm{kcal} / \mathrm{mole}$, therefore it will proceed 157 spontaneously; however, the activation energy is unknown. Together with the liberated $158 \mathrm{Na}$, water is produced, with the supply rate of water molecules being half the supply rate 159 of $\mathrm{Na}$ by this process. The solar wind protons are implanted into the regolith grains and 160 are neutralized there. The proton fluxes onto the surface are high and saturation of the 161 regolith grains with hydrogen can be safely assumed, which assures the availability of 162 hydrogen for the chemical reaction given above. In summary, hydrogen reacts with the 
163 sodium bearing rock, the $\mathrm{Na}$ atoms are liberated from their chemical bounds in the

164 crystal. Since the ion implantation is close to the surface, the atomic $\mathrm{Na}$ is produced near

165 the surface and will diffuse thermally to the surface. Atomic $\mathrm{Na}$ at the surface can be 166 easily released into the exosphere either by TD or by PSD (Yakshinskiy and Madey, 167 1999; 2004; Yakshinskiy et al., 2000). The process described in equation 1 causes also 168 the production of water. The global source rate fot $\mathrm{H}_{2} \mathrm{O}$ should be roughly half of that of $169 \mathrm{Na}$, even if the total exosphere content can be different because of different lifetimes. It is 170 worth noting that, recently, FIPS instrument on board MESSENGER has discovered 171 water group ions (Zurbuchen et al., 2008).

\subsection{Surface composition model}

The planetary surface was divided into $24 \times 48$ (latitude, longitude) surface elements.

175 Each surface element had a variable value for the sodium relative composition, $C(t)$,

176 which gives the numerical fraction of free sodium in the elementary surface with respect

177 to the total surface density. The time resolution of the simulation was 10 minutes; the 178 simulation lasted for a full Mercury's day (176 days). For each surface element, we 179 calculated the temporal evolution of $C(t)$, taking into account:

180 i) the planetary rotation and orbit;

181 ii) the solar wind proton precipitation;

iii) the photon-stimulated desorption;

183 iv) the thermal desorption;

$184 \quad v$ ) the photoionization of sodium.

185 At each step, we first calculated the position of each surface element with respect to 186 the Sun, taking into account the planetary rotation and orbital speed. The apparent 187 angular velocity of the Sun is assumed constant. In fact, we were far from the perihelion 188 where the apparent angular velocity has large variability (where it is retrograde for few 189 days).

190 Then, for each surface element we calculated the photon-stimulated desorption rate: 


$$
\Phi_{P S D}(t)=N C(t) \Phi_{\gamma} \sigma \cos (\alpha)=A C(t),(2)
$$

192 where $\Phi_{P S D}$ is the PSD neutral flux, $\Phi_{\gamma}$ is the relevant photon flux $\left(3 \times 10^{15} \mathrm{~cm}^{-2} \mathrm{~s}^{-1}\right.$ at 1 $193 \mathrm{AU}$; the flux is scaled as $\left.1 / r^{2}\right), \sigma$ is the PSD cross-section $\left(2 \times 10^{-20} \mathrm{~cm}^{2}\right.$, Yakshinskiy and 194 Madey, 1999), $N$ is the surface density $\left(7.5 \times 10^{14} \mathrm{~cm}^{-2}\right.$, Killen et al., 2001), $\alpha$ is the 195 instantaneous angle from the sub-solar point (for $\alpha>90^{\circ}, \Phi_{p s d}$ is obviously zero).

196 To calculate the value of $C$ at the equilibrium, we set $C(0)=0$ and calculate the value 197 of $C(t)$ at the next integration step, by solving the following differential equation:

$$
N \frac{d C(t)}{d t}=k \Phi_{P R E C}-\Phi_{P S D}(t)=k \Phi_{P R E C}-A C(t),(3)
$$

where $A$ is defined in Eq. $2 ; \Phi_{P R E C}$ is the flux of precipitating protons, and $k$ is the product of the overall process yield and the probability for the proton to interact with a

$201 \mathrm{Na}$ atom in the surface; by considering the fraction of $\mathrm{Na}$ bearing minerals (Feldspars) in the regolith (Wurz et al., 2008), here we have assumed that $k$ is about $5 \%$.

When a surface element is on the dayside, $C(t)$ raises or decreases exponentially, until 204 it reaches the equilibrium:

$$
\Phi_{P S D}=k \Phi_{P R E C} ;(4)
$$

206 the time-scale for the equilibrium is:

$$
T_{S C}=\left(\Phi_{\gamma} \cdot \sigma\right)^{-1}(5)
$$

208 which is of the order of one hour. When a surface element is on the night side, $C(t)$ 209 increases if $\Phi_{\text {prec }}>0$ (i.e., magnetospheric protons precipitate onto the surface) until this 210 surface element reaches the dawn terminator. Then, $C(t)$ is rapidly decreased by PSD 211 following Eq. 3. This leads to a maximum of concentration close to the dawn terminator, 212 and hence to a dawn-dusk asymmetry of $C$.

213 Note that the total amount of sputtered sodium particles has to be proportional (see 214 Eq. 4) to the total flux of proton precipitating onto the surface. Hence, the parameters $\Phi_{\gamma}$, 215 and $\sigma$ influence only on time scales of the simulated density, but they have very small 216 impact on the results at the equilibrium. 
Concerning thermal desorption, we find that particles released by TD always fall

218 down onto the surface because their velocities are much lower than the escape velocity.

219 Hence, TD does not contribute to the net flux from the surface. However, as outlined

220 below, the $\mathrm{Na}$ atoms fall back within an area of radius $<300 \mathrm{~km}$ (about $100 \mathrm{~km}$ on

221 average, in our simulation). Thus, thermal desorption will cause a smearing of the places

222 of Na release on the dayside. To include this effect in our simulation, we first estimated

223 the TD rate:

$$
\Phi_{T D}(t)=v N C(t) e^{-\frac{U_{T D}}{k_{B} T}},(6)
$$

225 where $\Phi_{T D}$ is the neutral flux, $v$ is the vibration frequency of the adsorbed atoms $\left(10^{13} \mathrm{~s}^{-1}\right.$, 226 Hunten et al., 1988), $N$ is again the regolith surface density, and $U_{T D}$ is the binding 227 energy. This last parameter has a big influence on the desorption rate, and has to be 228 evaluated carefully (Yakshinskiy et al., 2000). Yakshinskiy et al. (2000) determined an 229 energy range for $U_{T D}$ between 1.4 to $2.7 \mathrm{eV}$. In this study, however, we used an average 230 value for $U_{T D}=1.85 \mathrm{eV}$, like Leblanc and Johnson (2003). The local temperature of the 231 surface was assumed to vary according to (Leblanc and Johnson, 2003; Wurz and 232 Lammer, 2003):

$$
T(\alpha)=T_{n}+\left(T_{d}-T_{n}\right)[\cos (\alpha)]^{1 / 4}(7)
$$

234 where the sub-solar point temperature $\left(T_{d}\right)$ is scaled between $590 \mathrm{~K}$ and $725 \mathrm{~K}$ (Vilas, 235 1988) according to the planetary distance from the Sun. The night side temperature was 236 always $110 \mathrm{~K}$.

237 Then, for each surface element, we distributed the flux $\Phi_{T D}$ to a large number of test238 particles. We simulated their ballistic trajectories, starting with a Maxwell-Boltzmann 239 surface velocity distribution with the proper surface temperature. We then evaluated the 240 precipitation position and the lifetime of each test particle, and we redistributed the flux $241 \Phi_{T D}$ accordingly (decreased by photoionization, with lifetime $10^{4} \mathrm{~s}$ ). This latter flux can 242 be considered as an additional source in Eq. 3, while $\Phi_{T D}$ can be added to $\Phi_{P S D}$ in the 243 sink section of Eq. 3. Hence (as far as photoionization is negligible), the net effect is just 244 a time and spatial blurring of the solution of Eq. 3 . 
245 Our simulation lasted one complete Mercury day. However, if we take $C$ in a 246 reference frame fixed with respect to the Sun (i.e., MLT, Mercury Local Time), then $C$ 247 reaches a stable equilibrium after about half a rotation or less (i.e. tens of days). In Figure 2483 we show intermediate steps of the temporal evolution of parameter $C$ at different times.

249 After one hour, the dayside configuration is more or less stable. In Figure 4 a we show the 250 values of $\Phi_{P S D}$ at the equilibrium, in a MLT vs. latitude map, and, for comparison, the 251 proton precipitation flux (panel b). Since the flux in the dayside is basically proportional 252 to the proton flux (Eq. 4), the two panels are similar in that area. The dawn-dusk 253 asymmetry of the composition is responsible of the enhancement of $\Phi_{P S D}$ close to the 254 dawn terminator ( 6:00 MLT).

$255 \quad$ [Figures 3, 4] Monte-Carlo model. The energy distribution of the emitted $\mathrm{Na}$ atoms was extrapolated 260 (Johnson et al., 2002) by laboratory measurements of electron-stimulated desorption 261 (ESD) of adsorbed $\mathrm{Na}$ from amorphous ice. It was assumed that the electron energy has 262 little impact on the emitted neutral energy, and that PSD and ESD cause desorption of 263 atoms via similar electronic processes. Johnson et al. (2002) have found a good analytical 264 description of the energy spectrum using the following function:

$$
f(E)=\beta(1+\beta) \frac{E U^{\beta}}{(E+U)^{2+\beta}},(8)
$$

266 where $\beta$ is the shape parameter $(0.7$ for $\mathrm{Na})$ and $U$ is the characteristic energy, which is of 267 the order of $0.05 \mathrm{eV}$, for $\mathrm{Na}$. Since the maximum ejection energy should be lower than 268 the photon energy, we used a cut-off function (at about $10 \mathrm{eV}$ ) to eliminate the high269 energy tail of the function, which we consider unphysical. To evaluate the effect of the 270 source distribution function on the simulated exospheric density, we have also used a 271 different source function: 


$$
f(E)=\frac{\mathrm{E}}{\left(k_{B} T\right)^{2}} e^{\left(-E / k_{B} T\right)}(9)
$$

273 since other authors (Yakshinskiy and Madey, 1999, Leblanc and Johnson, 2003)

274 suggested that the energy spectrum has a Maxwellian Boltzmann flux distribution, with $T$ 275 of the order of 1000 to $1500 \mathrm{~K}$.

276 A large number $\left(>10^{6}\right)$ of test particle trajectories were simulated; a weight $w$ was 277 associated to each test particle to reproduce the flux in Eq. 2 (see details in Mura et al., 278 2007). The trajectories were calculated using the classical equations of motion, including 279 gravity and radiation pressure acceleration. The radiation pressure acceleration, for $\mathrm{Na}$, 280 can be up to $54 \%$ of the surface gravity, ranging between 20 and $200 \mathrm{~cm} \mathrm{~s}^{-2}$, being 281 function of the photon flux and of the amount of Doppler shift out of the Fraunhofer 282 features (Smyth and Marconi, 1995; Potter et al., 2002). Here we calculate this acceleration as in Leblanc and Johnson, (2003), i.e. we calculate the Doppler shift as a

284 function of each particle's velocity, and then we calculate the acceleration; on average, at $0.45 \mathrm{AU}$, this is $60 \mathrm{~cm} / \mathrm{s}^{2}$, in the anti-sunward direction.

We defined a 3D cubic accumulation grid extending between $-12 \mathrm{R}_{\mathrm{M}} \leq x \leq 3 \mathrm{R}_{\mathrm{M}}$ (30 steps) and $-2 \mathrm{R}_{\mathrm{M}} \leq y, z \leq 2 \mathrm{R}_{\mathrm{M}}$ (60 steps), with $\mathrm{R}_{\mathrm{M}}=2439 \mathrm{~km}$ being the radius of Mercury and $x$ pointing towards the Sun, $z$ pointing towards north pole (MSE reference frame). The grid has wider $x$ boundaries to enclose all the Sodium (see, for example, Potter et al., 2002). The Na density inside one grid cell was calculated taking into account the number

291 of test particles, the weight $w$ and the lifetime inside the cell. Finally, we integrated the 292 simulated Na density along the $x$ direction to obtain the tangential column density, to be 293 compared directly with the observations.

\section{Results and discussion}

\subsection{Model results}

To reproduce the observed tangential column density, we need to assume some values for the simulation parameters. As already mentioned, TD causes just a temporal and 
300 the simulations. Leblanc and Johnson (2003) arrived at the same conclusion when they 301 compared the residence time of sodium atoms related to TD, PSD and sputtering in 302 Mercury's upper surface versus the solar zenith angle. It was found that TD is the most 303 efficient desorption process at low zenith angles but at higher zenith angles PSD and 304 sputtering become more efficient.

305 One of the most important parameters to be considered is the energy $U$ (in Eq. 8), 306 which controls the energy distribution of the emitted sodium atoms. A best fit between 307 data and simulations to find the optimum value for $U$ is not easy; however, we found that 308 a good agreement between observations and simulation can be obtained by taking $U=$ $3090.086 \mathrm{eV}$. In Figure $5 \mathrm{a}$ the result of the simulated sodium tangential column density is 310 given. As it can be seen, the simulated data show the same features as the observed ones 311 (Figure 1), which are the dawn-dusk and the north-south asymmetry. In the simulation we 312 find that the maximum tangential column density is located at the limb in the north-dawn 313 region and has a value of about $6 \times 10^{10} \mathrm{~cm}^{-2}$.

[Figure 5]

Moreover, the apparent scale-height is similar to that in the observations (Figure 1).

319 a more energetic source distribution produces higher scale-heights. To evaluate how 320 sensitive our model is to the chosen energy distribution, we produced a similar 321 simulation, using Eq. 9 instead of Eq. 8, with $T=1000 \mathrm{~K}$. This energy distribution is 322 somewhat less energetic than the previous one (Mura et al., 2007); the result of the 323 simulation is shown in Figure 5b. There is a substantial resemblance between the two 324 simulations, and it is difficult to tell just by visual inspection which fits better with the 325 observations However, apparently the scale height of the first energy distribution (Eq. 8) 326 fits better with the observations.

327 More information on the energy distribution of the source can be obtained from the 328 observed velocity distribution. To study the velocity distribution of $\mathrm{Na}$ in the $x$ direction, 
329 a fourth dimension of the accumulation grid, consisting of 100 velocity steps from -10 to

$330+10 \mathrm{~km} / \mathrm{s}$, was included. In this way we were able to estimate the velocity distribution

331 shown in Figure 6. According to Schleicher et al. (2004), the observed velocity

332 distribution can be reproduced by a Gaussian function with a width $v_{t h}=1.6 \mathrm{~km} / \mathrm{s}$. As

333 shown in Figure 6, the simulation is able to reproduce this feature reasonably well using

334 the energy distribution of Eq. 8. The small secondary peak, visible at about $2 \mathrm{~km} / \mathrm{s}$ in the

335 figure, is probably due to the radiation pressure acceleration, which originates in the tail

336 up to few $\mathrm{R}_{\mathrm{M}}$ behind the planet. In fact, this is the value that can be obtained by simple

337 calculations assuming an acceleration of $-60 \mathrm{~cm} / \mathrm{s}^{2}$ and a tail size of about $3 \mathrm{R}_{\mathrm{M}}$ as

338 reported by Potter et al. (2002). For comparison, the velocity distribution obtained using

339 Eq. 9 (dashed line in Figure 6) substantially differs from the previous two. We concluded

340 that the source energy distribution is likely to be more energetic than a Gaussian at 1000 $341 \mathrm{~K}$

342 The effect of the radiation pressure acceleration on the observed column densities is 343 negligible; in fact, since this force acts parallel to the line of sight, the first order effect is 344 null. To evaluate the effect of the radiation pressure, we removed this force from the 345 model: the simulated tangential column densities were very similar, almost identical, to 346 those shown in Figure 5a; however, the fraction of escaping $\mathrm{Na}$ particles dropped down 347 by a factor 2 . We concluded that the radiation pressure acceleration, in the present 348 configuration, is responsible of about $50 \%$ of the $\mathrm{Na}$ escape rate.

\section{$349 \quad$ [Figure 6]}

350 Table 1 gives a summary of the observed and simulated physical quantities. The 351 tangential column densities are very close. However, the typical scale height in the model 352 calculations is higher than in the observations and therefore the total amount of $\mathrm{Na}$ in the 353 simulation is higher than that in the observations.

$354 \quad[$ Table 1]

355 The gardening rate at Mercury is estimated to cause a grain lifetime on the surface of 356 the order of $10^{4}-10^{5}$ years (Horz et al., 1991); a complete discussion about how this 357 lifetime implies diffusion-limited supply rates in the exosphere of Mercury can be found 358 in Killen et al. (2004). These authors found that a supply rate up to $10^{7} \mathrm{~cm}^{-2} \mathrm{~s}^{-1}$ is 
359 consistent with a diffusion limited supply of Na. For comparison, in our study, the 360 average PSD flux on the surface was found to be of the order of $10^{7} \mathrm{~cm}^{-2} \mathrm{~s}^{-1}$, but the local

361 PSD rate (see Figure 4) was higher in the areas of intense proton precipitation. This is 362 consistent with the results of Killen et al., (2004) when saying that higher supply rates

363 can be the result of ion-enhanced diffusion.

\subsection{Ion sputtering}

366 The presence of the two maxima observed at high latitude is a hint for the presence of 367 a space weathering process, such as ion sputtering. Hence, we simulated the $\mathrm{Na}$ 368 exospheric density resulting from this process. We assumed that the proton precipitation 369 flux was the same as shown in Figure 4b. Each impinging proton has some probability 370 (yield, $Y$ ) to extract a neutral sodium atom from the surface; this probability is related to 371 the proton energy (Lammer et al., 2003); we have assumed an averaged value of $10 \%$ for 372 simplicity. Other solar wind components, like $\alpha$ particles or high-charge-state particles, 373 produce a comparable amount of ion sputtering (Johnson and Baragiola, 1991). 374 Assuming that their precipitation patterns are similar to that of protons, we assumed a 375 weighted yield of $20 \%$ as an upper limit for sputtering. The resulting $\mathrm{Na}$ flux is hence:

$$
\Phi_{I S}=Y_{c} \Phi_{P R E C}
$$

where $c$ is the surface abundance of sodium in the surface. While thermal and photon378 stimulated desorption act on the extreme surface (a few monolayers, where the 379 concentration of $\mathrm{Na}$ should be calculated as in Section 3.2), ion-sputtering extracts 380 sodium from deeper and in a stoichiometric way. Hence, we assumed $c$ uniform all over 381 the surface and equal to $0.5 \%$ (Goettel, 1988).

382 The energy distribution of the ejecta can be simulated using the following function 383 (Siegmund, 1961; Betz and Wien, 1994):

$$
f_{S}\left(E_{e}\right) \propto \frac{E_{e}}{\left(E_{e}+E_{b}\right)^{3}}\left[1-\left(\frac{E_{e}+E_{b}}{E_{i}} \frac{\left(m_{H}+m_{N a}\right)^{2}}{4 m_{H} m_{N a}}\right)^{\frac{1}{2}}\right],
$$


385 where $E_{e}$ is the ejection energy, $E_{i}$ is the proton energy, $E_{b}$ is the binding energy (here 2

$386 \mathrm{eV}$, as in Lammer et al., 2003). We used this as an input for the exospheric model, then

387 we integrated the result to obtain the tangential column densities to be compared with the

388 observations. The result is shown in Figure 7a. There are at least three discrepancies

389 between this simulation and the observations:

390 - the tangential column densities are about two orders of magnitude lower than those

391 observed; in fact, for each proton, only $0.05 \%$ of $\mathrm{Na}$ is sputtered, i.e. released into the $392 \quad$ exosphere $(Y$ times $c)$.

393 - the simulated scale-heights are considerably larger than those observed; in fact the 394 energy distribution in Eq. (11) is more energetic than that in Eq. (8);

395 - there is no evident dawn-dusk asymmetry.

396 In summary, ion sputtering, alone, is not able to explain the observed features. 397 Moreover, since the contribution to the total column densities is only about $1 \%$ of the 398 observed values, which is consistent with the results from Wurz et al. (2007), it is 399 possible to neglect its contribution in the particular configuration of the present study. On 400 the other hand, since the process is more energetic, most of sputtered $\mathrm{Na}$ are either 401 photoionized (due to longer ballistic time) or lost (due to Jeans escape). Hence, this 402 process must be taken into account while simulating the Na tail, as in section 4.4.

\subsection{Meteoritic and micrometeoritic impact vaporization}

404 Mercury is exposed to the constant precipitation of particles of small sizes $(<100 \mu \mathrm{m})$, 405 impacting the surface at a mean velocity of $20 \mathrm{~km} / \mathrm{s}$ (Cintala, 1992), churning the regolith 406 and vaporizing the surface. Larger objects impact the surface as well, causing local 407 enhancement of the sodium exospheric density (Mangano et al., 2007), but the 408 contribution by these meteorites to the global Hermean exosphere is considered to be 409 negligible (Killen et al., 2007). Morgan et al. (1988) reported an average value of $2 \times 10^{5}$ $410\left(\mathrm{~cm}^{-2} \mathrm{~s}^{-1}\right)$ released $\mathrm{Na}$ at aphelion, a factor 2 higher than the value reported by Cintala 411 (1992) (see Killen et al., 2007 for a detailed discussion). The first value, which we have 412 used here as a worst case, is a factor 100 lower than our averaged PSD flux. If we assume 413 that the ejecta have a thermal velocity distribution at about $2500 \mathrm{~K}$ (Killen et al., 2007), 
414 and that the precipitating particles are uniformly distributed over the surface, we obtain

415 the simulated $\mathrm{Na}$ tangential column densities of Figure $7 \mathrm{~b}$. Obviously, the precipitation

416 flux could be not uniform on the surface and so our estimation of the density due to

417 impact vaporization is probably locally inaccurate to a factor of two (Killen et al., 2004);

418 however, this simulation shows that, at a first order of approximation, we can discard

419 impact vaporization as a main $\mathrm{Na}$ source in this particular observing configuration.

\subsection{The sodium tail}

The presence of a sodium tail, extending in the anti-sunward direction, has been first observed by Potter et al. (2002). Since this is an important feature of the sodium

423 exosphere, it should be taken into account by any model. In this section we discuss the

424 formation of the $\mathrm{Na}$ tail within our present model. The sodium tail is formed by those $\mathrm{Na}$ 425 atom whose ejection energy is higher than the gravitational escape energy $(2.07 \mathrm{eV})$, or

426 by that atoms that can overcome the gravitational field thanks to a partial contribution of 427 the radiation pressure acceleration. These particles are accelerated by the radiation 428 pressure in the anti-sunward direction. In addition, photoionized $\mathrm{Na}$ can be picked-up by 429 the solar wind. The radiation pressure acceleration during the observation time, at $150^{\circ}$ 430 anomaly angle, was about $60 \mathrm{~cm} \mathrm{~s}^{-2}$, well below the maximum $\left(200 \mathrm{~cm} \mathrm{~s}^{-1}\right)$, which occurs 431 at about $60^{\circ}$ and $300^{\circ}$ anomaly angle (Smyth and Marconi, 1995; Potter et al., 2002). The 432 relation between the solar radiation pressure and the tail formation has been recently 433 investigated by Potter and Killen (2008). According to these authors, the tail is not 434 detectable from Earth with a radiation pressure below about $100 \mathrm{~cm} \mathrm{~s}^{-2}$. In our case, the 435 acceleration was lower $\left(60 \mathrm{~cm} \mathrm{~s}^{-2}\right)$, but the proton precipitation was very intense, due to 436 the extreme solar wind conditions (see section 3). Hence, in our case we should obtain a 437 very faint tail. To simulate it, we used all the four processes described previously as a 438 source for our exospheric model; the PSD source function is that given in Eq. 8. The 439 result is shown in Figure 8. Such a tail is very faint and probably hard to observe from 440 Earth; in fact, in the nightside, the densities are 2 to 4 orders of magnitude lower than 441 those in the dayside. The contribution of thermal desorption is negligible, since only $3 \%$ 442 of the desorbed particles do not re-impact onto the surface, and only due to 443 photoionization. Also impact vaporization does not contribute significantly, since only $44410 \%$ of the ejected particles are able to escape, mostly due to photoionization. The 
445 fraction of particles desorbed due to photon-stimulation that escape was found to be 446 between $1 \%$ and $10 \%$, depending on the source function we used, while $50 \%$ of the ion-

447 sputtered particles are able to escape. However, since surface flux for PSD is higher, both 448 these two processes contribute significantly to the formation of the sodium tail. A 449 summary of these values can be found in table 2. We conclude that, in general, our model 450 is consistent with the existence of a sodium tail.

$451 \quad$ [Table 2]

452 Recently, the sodium tail has been observed during the first MESSENGER flyby 453 (McClintock et al., 2008). These authors have found that, at $2 \mathrm{R}_{\mathrm{M}}$ down the tail, the 454 traverse column density was $\sim 3.4 \times 10^{9}$ atoms $\mathrm{cm}^{-2}$. In our simulation, we found, for the 455 same quantity, a value slightly less than $10^{9} \mathrm{~cm}^{-2}$. Even if a detailed comparison is 456 beyond the scope of this study, we note that there is no contradiction between these 457 values. In fact, during those MESSENGER observations, the radiation pressure was close 458 to its maximum, i.e. three times higher than that assumed in this study.

5 Model limitations

460 A critical hypothesis in our model is to assume that the proton precipitation flux (on 461 the night side) is constant over a very long time-scale ( weeks), which is clearly a major 462 simplification. However, as discussed in Section 3.2, the time-scale for the equilibrium 463 between proton fluxes and PSD fluxes is very short on the dayside (about one hour). 464 Therefore, we propose the following explanation. The north-south asymmetry is due to 465 the proton precipitation on the dayside, which rapidly results in an enhancement of the 466 Na density in the high latitude regions (by means of the combined mechanism described 467 in Section 3.1). The dawn-dusk asymmetry, on the other hand, is caused by the planetary 468 rotation, which is always in the same direction (except a few days of apparent retrograde 469 Sun motion), and by the presence of some magnetospheric proton precipitation on the 470 night side; such a precipitation is predicted for most of the IMF conditions (see, for 471 example, Kallio et al., 2003, or Mura et al., 2005). Hence, in this first study we did not 472 perform a detailed estimation of the proton circulation and precipitation; moreover, the 473 TD effect causes a smearing of the surface composition, so that a detailed map of the 474 proton precipitation is not needed. 


\section{Summary and Conclusions}

477 We presented a model for the formation of Mercury's sodium exosphere as it was 478 observed during the Mercury transit on May 7, 2003, involving two steps for the release 479 of $\mathrm{Na}$ atoms from the surface. The first step is the liberation of $\mathrm{Na}$ from the chemical 480 environment of the mineral by implantation of energetic hydrogen and subsequent 481 chemical reaction. The second step is photon-stimulated desorption of $\mathrm{Na}$ into the 482 exosphere. Thermal desorption only smears the location where the $\mathrm{Na}$ atoms are released. 483 For the simulation of this two-step process, a 3D exospheric model was adapted 484 accordingly.

485 Comparison of our model results with measurements by Schleicher et al. (2004) show 486 good qualitative agreement in the structure of Mercury's sodium exosphere. Our model 487 reproduces the high latitude features, and the asymmetries in the north-south and in the 488 dawn-dusk direction. Concerning the quantitative comparison, the simulated $\mathrm{Na}$ total 489 amount is of the same order of magnitude as that derived from observation. There are, 490 however, at least 3 parameters that control this value:

491 1) The scale height. The parameter $U$ in Eq. 8 controls the energy distribution of the 492 PDS emitted $\mathrm{Na}$, and hence the scale-height of the simulated exosphere. A larger scale493 height in the model causes the dayside population (which is the most relevant one) in the 494 simulations to rise above the planetary limb and therefore to become more visible from 495 the night side. Hence, the value of $U$ is also related to the overall $\mathrm{Na}$ amount. Since the 496 scale-height of the simulation is higher than that of the observation, this may suggest that 497 a lower value for $U$ should be used. We have also performed some simulations, using a 498 simple Gaussian distribution function (with temperature of $1000 \mathrm{~K}$ ), instead of Eq. 8. 499 Even if the simulated densities (Figure 5b) and scale-heights were very similar to the 500 observed ones, such energy distribution failed to explain the measured velocity spread.

501 2) The proton flux. The simulated densities are proportional to the total precipitation 502 rate, and the shape depends on that of the proton flux onto the surface. In particular, the 503 proton precipitation onto the nightside, thanks to the rotation of the planet, is responsible 504 for the formation of the dawn dusk asymmetry. In addition to proton precipitation other 
505 magnetospheric ions will precipitate as well. For example, $\mathrm{Na}^{+}$precipitation occur 506 nightside (see Delcourt et al., 2003), which will increase the surface weathering effects.

507 In fact, according to Potter (1995), in the case of chemical sputtering caused by $\mathrm{Na}^{+}$, the 508 yield should be higher. In any case, the inclusion of any other precipitation or 509 replenishing process occurring on the nightside, which we have neglected in this first 510 simulation, will cause an enhancement of the simulated dawn-dusk asymmetry, and, in 511 fact, the dawn-dusk asymmetry is a somewhat more evident in the observations than in 512 the model. The effect of other ion precipitation on the nightside, as well as impact 513 vaporization, not included in the present simulation, can be estimated using the model. In 514 fact, adding an hypothetical, uniform value of Sodium $2 \times 10^{6} \mathrm{~cm}^{-2} \mathrm{~s}^{-1}$ lead to a tangential 515 column density of $4 \times 10^{10} \mathrm{~cm}^{-2}$, which is what is observed close to the dawn terminator. 516 In this way, it is possible to estimate the upper limit of the $\mathrm{Na}$ replenishment process that 517 can occur in the nightside as $2 \times 10^{6} \mathrm{~cm}^{-2} \mathrm{~s}^{-1}$.

518 3) The process yield. Here, we have assumed that the averaged process yield is $5 \%$, 519 and the simulated densities scale linearly with this parameter. Recently, Sarantos et al., 520 (2008) have studied the influence of ion-enhanced PSD at the Moon, following the 521 mechanism proposed by Potter (2000); the mechanism is somewhat different from that 522 proposed here, but the global result is the same. These authors observed that the ion flux 523 increased to $3 \times 10^{7} \mathrm{~cm}^{-2} \mathrm{~s}^{-1}$ during the passage of the Moon in the Earth's magnetotail, 524 and as a result, the PSD rate was higher then expected, and of the order of $2 \times 10^{5} \mathrm{~cm}^{-2} \mathrm{~s}^{-1}$. 525 This would result in an averaged yield of the order of $1 \%$, which is in good agreement 526 with our assumption, also because one could expect that the yield of the process may 527 depend on the ion energy ranges, that may differ from the Moon to Mercury.

528 Since the PSD release $\mathrm{Na}$ flux is proportional to the proton precipitation, one could 529 expect that, on the dayside (which is not visible in the observation) the Na exospheric 530 densities should resemble the proton flux in Figure 4b. As shown in Figure 8, however, 531 this is not the case. In fact, on the dayside also the Na population from thermal desorption 532 is present. Moreover, in the case of a non-uniform surface source, Mura et al., (2007) 533 have estimated that the characteristic horizontal scale lengths of the exosphere are about a 534 factor 2 longer than that of the source, due to the ballistic trajectories of the particles. 
535 Another effect, not yet included in this model, can be the Na release due to thermal

536 diffusion, that should be maximal in the sub-solar point region, between our two

537 precipitation maxima (Killen et al., 2004).

538 The Na density in the tail predicted by our model is also in substantial agreement with 539 that observed during the first MESSENGER flyby (McClintock et al., 2008). Moreover,

540 chemical sputtering of $\mathrm{Na}$ by protons causes also the production of water, and it is worth

541 noting that the FIPS instrument on board MESSENGER has discovered such water group

542 ions in the exosphere of Mercury (Zurbuchen et al., 2008).

543 In a separate simulation run we have tried to simulate the $\mathrm{Na}$ density in a different 544 way, by assuming that the proton precipitation causes only direct ion-sputtering of 545 surface sodium. However, the simulated scale-heights were larger and the tangential 546 column densities were about 100 times lower than those observed, because ion-sputtering 547 has a lower yield (5\%) and a more energetic velocity distribution. On the other hand, it is 548 difficult to explain North-South asymmetries without including plasma precipitation 549 effects. We also simulated the $\mathrm{Na}$ exospheric density arising from impact vaporization; 550 also in this case, the tangential column densities were lower by a factor 100 . Presently, it 551 is therefore not possible to explain these observations without including a process that 552 combines ion-precipitation and PSD via the chemical alteration of the surface induced by 553 ion impact.

554

555

556 557 561

\section{Appendix}

From the detected excess absorption, densities of the sodium atoms in Mercury's exosphere could be derived using the following equations:

$$
\begin{gathered}
W_{\lambda}(h, \varphi)=\int_{-\infty}^{+\infty} \frac{\Delta I_{\lambda}(h, \varphi)}{I_{\lambda}}(\mathrm{A} 1) \\
\frac{\Delta I_{\lambda}(h, \varphi)}{I_{\lambda}}=1-\exp \left(-\tau_{\lambda}\right)(\mathrm{A} 2) \\
\tau_{\lambda}=\kappa_{\lambda}^{(A)} n(h, \varphi)(\mathrm{A} 3) \\
\kappa_{\lambda}^{(A)}=\kappa_{0}^{(A)} \frac{1}{\sqrt{\pi} \Delta \lambda_{D}} \exp \left(-\frac{\lambda}{\Delta \lambda_{D}}\right)^{2}
\end{gathered}
$$


562 Here, $W_{\lambda}(h, \varphi)$ is the equivalent width of the absorption excess $\Delta I_{\lambda}$, observed at

563 location $h$ above the limb at azimuth $\varphi . I_{\lambda}$ is the solar background intensity (that is the

564 undisturbed solar $\mathrm{D}_{2}$ line profile). $\kappa_{0}^{(A)}$ is the atomic absorption coefficient at line center of

565 the resonance line, $\Delta \lambda_{D}$ the Doppler width of the excess absorption. By inserting Eq. A2-

566 A4 into Eq. A1 one gets the relation between equivalent width and $n$, the column density

567 along the line of sight, which is nearly linear, because the excess absorption is nearly

568 optically thin. Using this relation, the tangential column densities displayed in Figure 1

569 have been derived from the observed equivalent widths; for the Doppler width the value

570 measured from the observed averaged profile of the excess absorption were used.

\section{$572 \quad$ Acknowledgments}

573 This work was supported by the Italian Space Agency (ASI). A. Mura and H. I. M.

574 Lichtenegger also acknowledge support from the EU funded Europlanet project due to its

575 N5 activity. We also thank the ACE SWEPAM instrument team and the ACE science 576 Center for providing ACE data. 
Betz, G., and K. Wien. Energy and angular distributions of sputtered particles, Int. J. of Mass Spectrometry and ion processes, 140, 1-110, 1994.

Broadfoot A.L., D.E. Shemanky, and S. Kumar, Mariner 10: Mercury atmosphere, Geophys. Res. Lett., 3, $577,1976$.

Cintala, M. J., 1992. Impact-induced thermal effects in the lunar and mercurian regoliths. J. Geophys. Res. 97, 947-973.

Delcourt, D. C., S. Grimald, F. Leblanc, J.-J. Berthelier, A. Millilo, A. Mura, S. Orsini, and T. E. Moore, A quantitative model of the planetary Na+ contribution to Mercury's magnetosphere, Ann. Geophys., 21, 1723, 2003.

Goettel, K.A., Present bounds on the bulk composition of Mercury: implications for planetary formation processes. In: Vilas, F., Chapman, C.R., Matthews, M.S. (Eds.), Mercury. Univ. of Arizona Press, Tucson, pp. 613-621, 1988.

Horz, F., Grieve, R., Heiken, G., Spudis, P., Binder, A., Lunar surface processes. In: Heiken, G., Vaniman, D.T., French, B.M. (Eds.), Lunar Sourcebook. Cambridge Univ. Press, Cambridge, pp. 61., 1991

Hunten, D.M., and A.L. Sprague, Origin and character of the lunar and mercurian atmospheres, Adv. Space

595 Hunten, D.M., T.H. Morgan, and D.E. Shemansky, The Mercury Atmosphere, In: Vilas, F., Chapman, C.R., Matthews, M.S. (Eds.), Mercury. Univ. of Arizona Press, Tucson, pp. 613-621, 1988.

Johnson, R.E., and R. Baragiola, Lunar surface: sputtering and secondary ion mass spectrometry. Geophys. Res. Lett., 18, 11, 2169-2172, 1991.

Johnson, R.E., Leblanc, F., Yakshinskiy, B.V., Madey, T.E. Energy distributions for desorption of sodium and potassium from ice: the $\mathrm{Na} / \mathrm{K}$ ratio at Europa. Icarus 156, 136-142, 2002.

601 Kallio, E. and P. Janhunen, Solar wind and magnetospheric ion impact on Mercury's surface Geophys. Res. Lett., Vol. 30, No. 17, 1877, 2003.

Killen, R.M., A. Potter, A. Fitzsimmons, T.H. Morgan, Sodium D2 line profiles: clues to the temperature 604 structure of Mercury's exosphere. Planet. and Space Sci., 47 1449-1458, 1999.

Killen, R. M., Potter, A. E., Reiff, P. H., Sarantos, M., Jackson,B. V., Hick, P., and Giles, B. L.: Evidence 606 for space weather at Mercury, J. Geophys. Res., 106, 20 509-20 526, 2001.

Killen, R.M., M. Sarantos, A.E. Potter, and P. Reiff, Source rates and ion recycling rates for Na and K in Mercury's atmosphere, Icarus 171 1-19, 2004. 
Killen, R., G. Cremonese, H. Lammer, S. Orsini, A. E. Potter, A. L. Sprague, P. Wurz, M. L. Khodachenko, H. I. M. Lichtenegger, A. Milillo and A. Mura, Processes that Promote and Deplete the Exosphere of Mercury, Space Science Reviews , 132, 433-509, 2007.

Lammer, H., Wurz, P., Patel, M.R., Killen, R., Kolb, C., Massetti, S., Orsini, S., Milillo, A., The variability of Mercury's exosphere by particle and radiation induced surface release process. Icarus 166, 238$247,2003$.

Leblanc F. and Johnson, R.E., Mercury’s sodium exosphere, Icarus, 164, 261-281, 2003.

Luhmann, J. G. and Friesen, L. M.: A simple model of the magnetosphere, J. Geophys. Res., 84, 44054408, 1979.

McClintock W. E., E. T. Bradley, R. J. Vervack Jr., R. M. Killen, A. L. Sprague, N. R. Izenberg, S. C. Solomon, Mercury's Exosphere: Observations During MESSENGER's First Mercury Flyby, 92, 321 Science, 2008

Madey, T.E., Yakshinskiy, B.V., Ageev, V.N., Johnson, R.E., Desorption of alkali atoms and ions from oxide surfaces: relevance to origins of $\mathrm{Na}$ and $\mathrm{K}$ in atmospheres of Mercury and the Moon. J. Geophys. Res. 103, 5873-5887, 1998.

V. Mangano, A. Milillo, A. Mura, S. Orsini, E. DeAngelis, A.M. DiLellis, and P. Wurz, The contribution of impact-generated vapour to the hermean atmosphere, Planet. Space Sci. 55(11), 1541-1556, 2007.

McGrath, M.A., Johnson, R.E., and Lanzerotti, L.J., Sputtering of sodium on the planet Mercury, Nature, 323, 694-696, 1986.

Morgan, T.H., H.A. Zook, A.E. Potter, Impact-driven supply of sodium and potassium in the atmosphere of Mercury. Icarus 74, 156-170,1988.

Mura, A., S. Orsini, A. Milillo, D. Delcourt, S. Massetti. Dayside H+ circulation at Mercury and neutral particle emission. 175, 305-319, 2005.

Mura, A., A. Milillo, S. Orsini, S. Massetti, Numerical and analytical model of Mercury's exosphere: Dependence on surface and external conditions, Planet. Space Sci., 2007.

Potter, A.E., and T.H. Morgan. Discovery of Na in the atmosphere of Mercury, Science 229, 651-653, 1985.

Potter, A.E., and T.H. Morgan. Evidence for suprathermal sodium on Mercury. Adv. Space Res. 19, 15711576. 1997.

Potter, A. E., R. M. Killen, and T. H. Morgan, Variation of lunar sodium during passage of the Moon through the Earth's magnetotail, J. Geophys. Res., 105, 15,073- 15,084, 2000.

Potter, A., Killen, R.M., Morgan, T.H., The sodium tail of Mercury, Meteor. Planet. Sci. 37 (9), 1165$1172,2002$. 
Potter, A., and R.M. Killen, Observations of the sodium tail of Mercury, Icarus 194, 1-12, 2008.

Sarantos, M., Reiff, P.H., Hill, T.W., Killen, R., Urquhart, A.L., A . . -interconnected magnetosphere model for Mercury. Planet. Space Sci. 49, 1629-1635, 2001.

Sarantos, M., R. M. Killen, A. S. Sharma, and J.A. Slavin. Correlation between Lunar Prospector measurements and the Lunar Exosphere during passage through the Earth's magnetosphere, Geophys. Res. Lett. 35, L04105, 2008.

Schleicher, H., G. Wiedemann, H. Wöhl, T. Berkefeld, and D. Soltau, Detection of neutral sodium above Mercury during the transit on 2003 May 7. Astronomy and Astrophysics, 425, 1119-1124, 2004.

Sigmund, P., Theory of sputtering. I. Sputtering yield of amorphous and polycrystalline targets. Phys. Rev. 184, 383-416, 1969.

Smyth, W. H., and M. L. Marconi, Theoretical overview and modeling of the sodium and potassium atmospheres of Mercury. Astrophys. J. 441: 839-864, 1995.

Sprague, A.L., Mercury's atmospheric bright spots and potassium variations: A possible cause, Jou. Geophys. Res. 97 18257-18264, 1992.

Vilas, F., Surface composition of Mercury from reflectance spectrophotometry. In: Vilas, F., Chapman, C.R., Matthews, M.S. (Eds.), Mercury. Univ. of Arizona Press, Tucson, pp. 59-76, 1988

Wurz, P., and H. Lammer, Monte-Carlo Simulation of Mercury's Exosphere, Icarus, 164 (1), 1-13, 2003.

Wurz, P., U. Rohner, J. A. Whitby, C. Kolb, H. Lammer, P. Dobnikar, and J. A. Martin-Fernandez (2007), The lunar exosphere: The sputtering contribution, Icarus, 191, 486-496, 2007.

Wurz, P., J. Whitby, U. Rohner, J.A. Martin-Fernandez, H. Lammer, and C. Kolb, "The Sputter Contribution to Mercury's Exopshere", Icarus, in preparation, 2008.

Yakshinskiy, B. V. and T. E. Madey, Photon-stimulated desorption as a substantial source of sodium in the lunar atmosphere, Nature 400, 642 - 644, 1999.

Yakshinskiy, B.V., Madey, T.E., Ageev, V.N., Thermal desorption of sodium atoms from thin SiO2 films. Surface Rev. Lett. 7, 75-87, 2000.

Yakshinskiy, B.V., Madey, T.E., Photon-stimulated desorption of Na from a lunar sample: temperaturedependent effects. Icarus, 168, 53-59, 2004.

T. H. Zurbuchen, J. M. Raines, G. Gloeckler, S. M. Krimigis, J.A. Slavin, Patrick L. Koehn, R. M. Killen, A. L. Sprague, R. L. McNutt Jr., S. C. Solomon, MESSENGER Observations of the Composition of Mercury's Ionized Exosphere and Plasma Environment, 90-92, 321, Science, 2008 
Table 1. Summary of observed and simulated physical quantities

\begin{tabular}{|c|c|c|}
\hline Parameter & Observations & Simulation \\
\hline Tan. col. dens. (max) & $7 \times 10^{10} \mathrm{~cm}^{-2}$ & $5 \cdot \times 10^{10} \mathrm{~cm}^{-2}$ \\
\hline Total amount & $4 \times 10^{27}$ & $5 \times 10^{27}$ \\
\hline Scale height & 200 to $500 \mathrm{~km}$ & $\sim 1000 \mathrm{~km}$ \\
\hline
\end{tabular}


Table 2. Loss rates

\begin{tabular}{|c|c|c|}
\hline Parameter & Jeans escape (\%) & Photoionization (\%) \\
\hline Photon Stimulated Desorption & $5 \%$ & $25 \%$ \\
\hline Thermal Desorption & $<1 \%$ & $3 \%$ \\
\hline Impact Vaporization & $2 \%$ & $10 \%$ \\
\hline Ion Sputtering & $45 \%$ & $45 \%$ \\
\hline
\end{tabular}




\section{Figure captions}

Fig. 1: Observed Na tangential column density during the Mercury transit of May 7, 2003. Data are from Schleicher et al. (2004). $y$ and $z$ axes are orientated according to the MSE frame (see text), i.e., $z$ is positive towards north and $y$ is positive towards dusk.

Fig. 2: Measured by ACE, during May 7-9, 2003, distance from spacecraft to Earth, components of magnetic field, solar wind proton speed and density (http://www.srl.caltech.edu/ACE/ASC/level2/index.html).

Fig. 3: Simulated temporal evolution of parameter $C(t)$. The pink line is a meridian line, fixed on the surface.

Fig. 4: Panel a: Simulated Sodium flux due to PSD. The flux in the dayside (8:00 to 16:00 MLT) is basically equal to the $\mathrm{H}+$ flux (see Eq. 4). The flux close to the dawn terminator (6:00) is enhanced because the Na composition in the night side is enriched by the proton precipitation. Panel b: $\mathrm{H}^{+}$precipitation flux.

Fig. 5. Panel a:Simulated Na tangential column density. Panel b: idem, but using the source distribution function in Eq. 9.

Fig. 6. Parallel $(x)$ velocity distribution of the simulated particles (dotted line). The observed velocity distribution can be reproduced by a Gaussian function with $v$ th $=1.6$ $\mathrm{km} / \mathrm{s}$ (solid line). The dashed line shows the simulated velocity distribution using Eq. 9. The small secondary peaks visible at about $2 \mathrm{~km} / \mathrm{s}$ are due to radiation pressure acceleration.

Fig. 7. Panel a: Simulated Na tangential column density, for Ion sputtering. Panel b: idem, but for impact vaporization.

Fig. 8. Simulated Na density in the $x z$ plane. Sun is to the right. 


\section{ACCEPTED MANUSCRIPT}

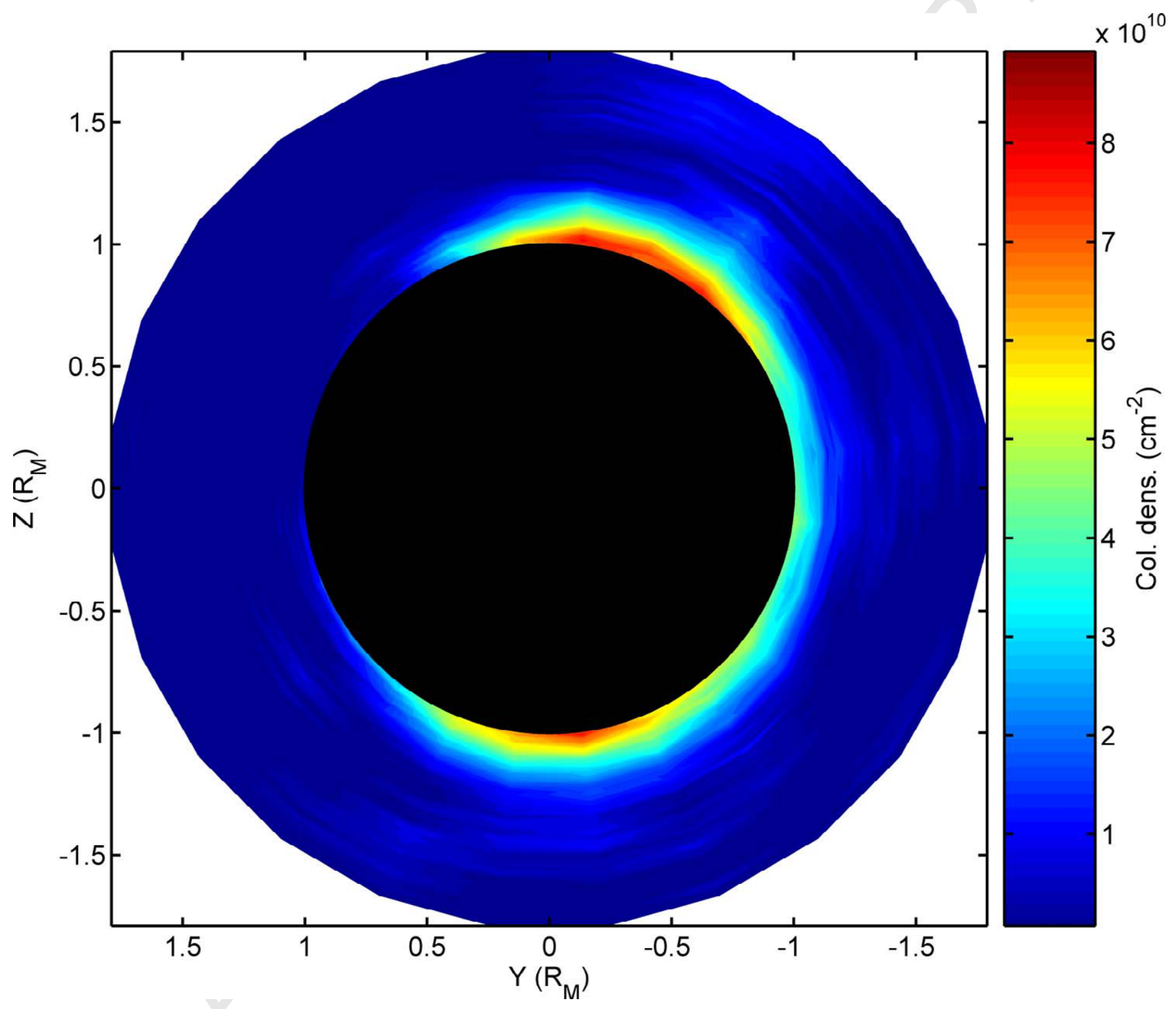




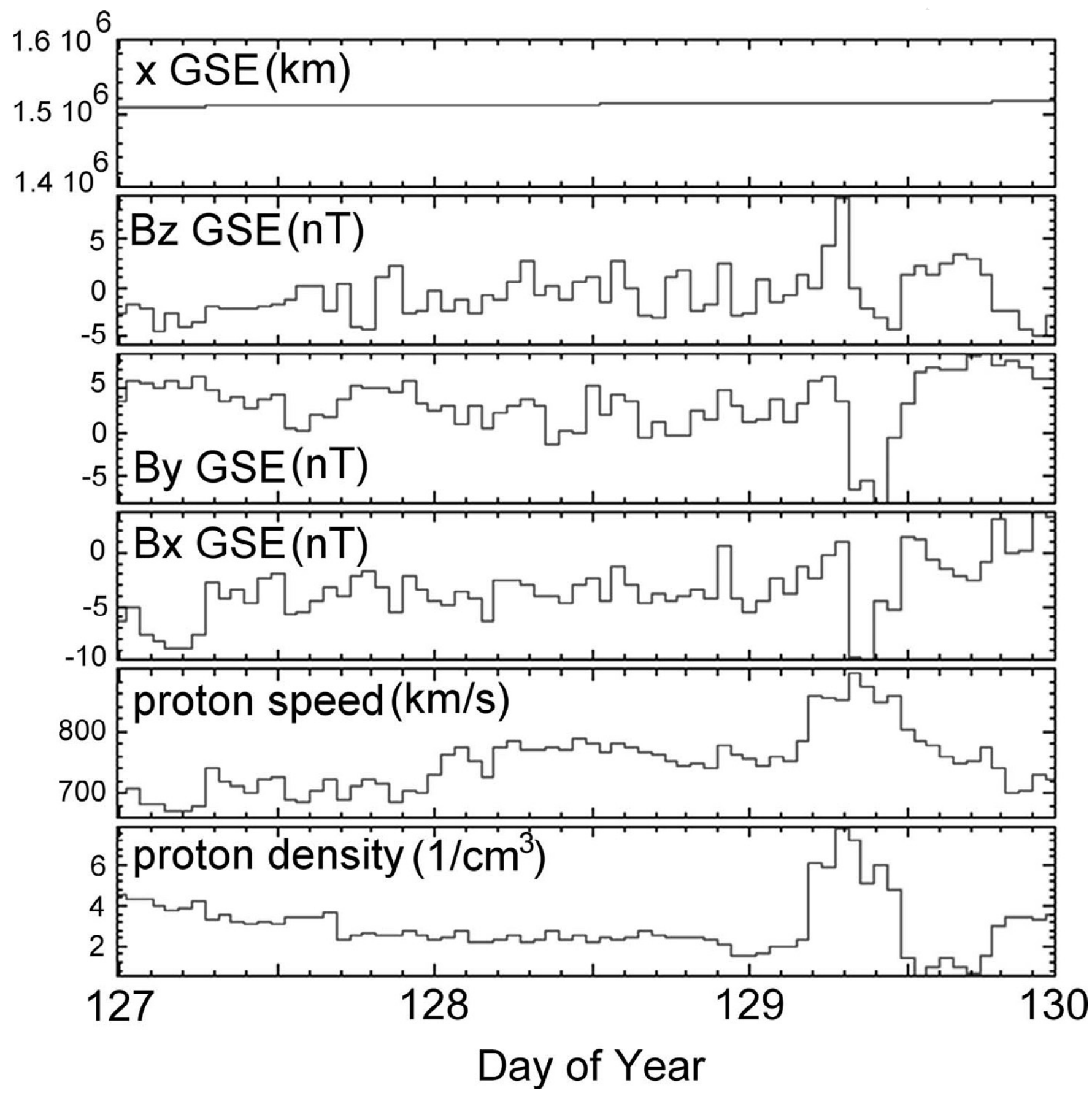



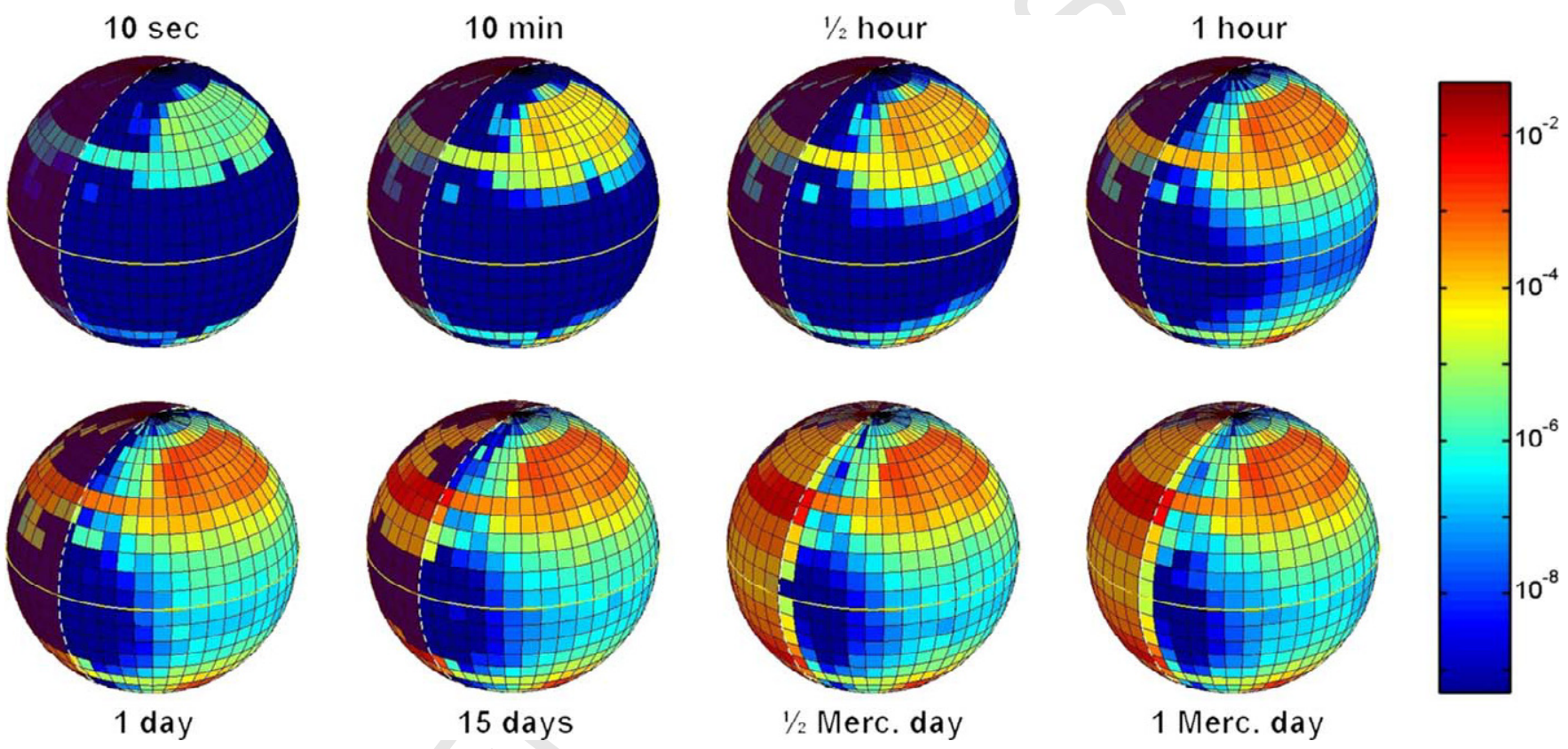
A
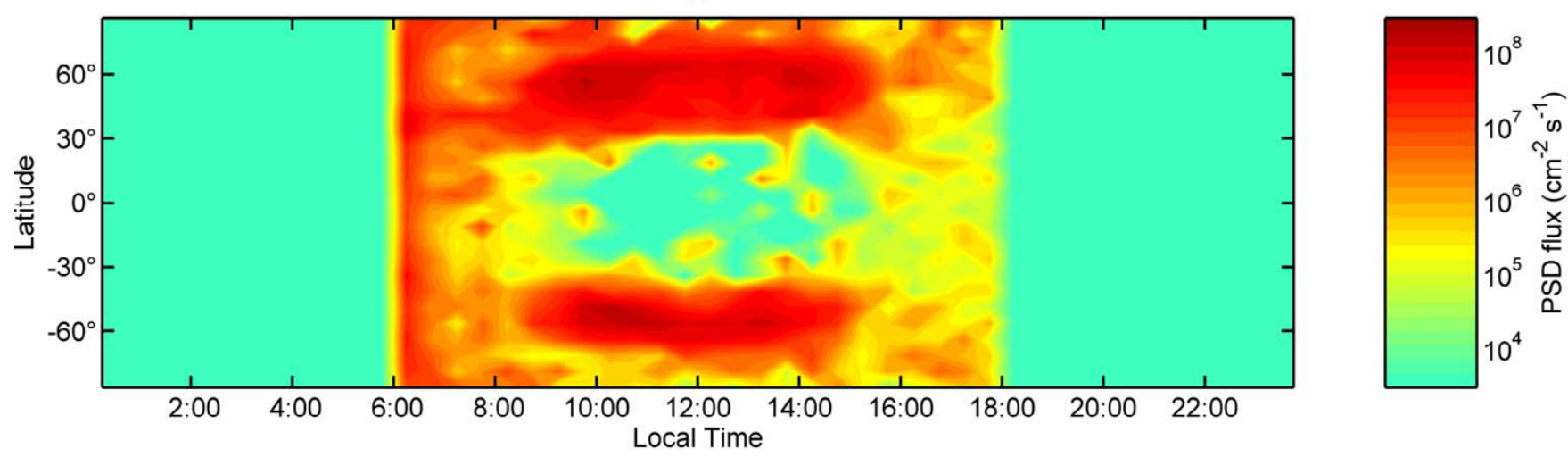

B
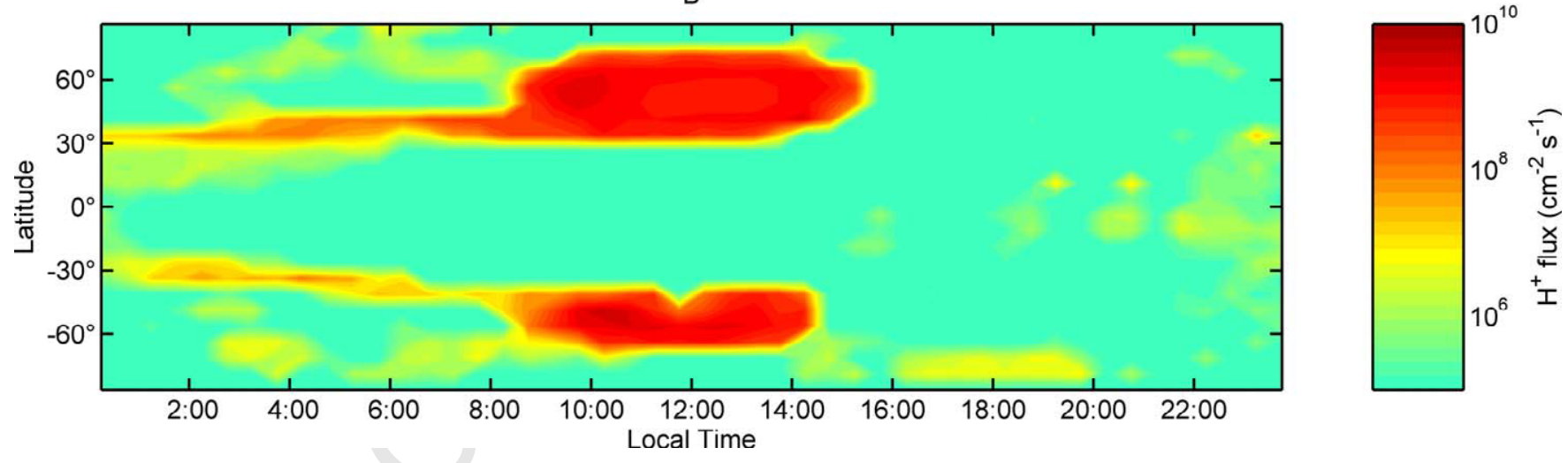


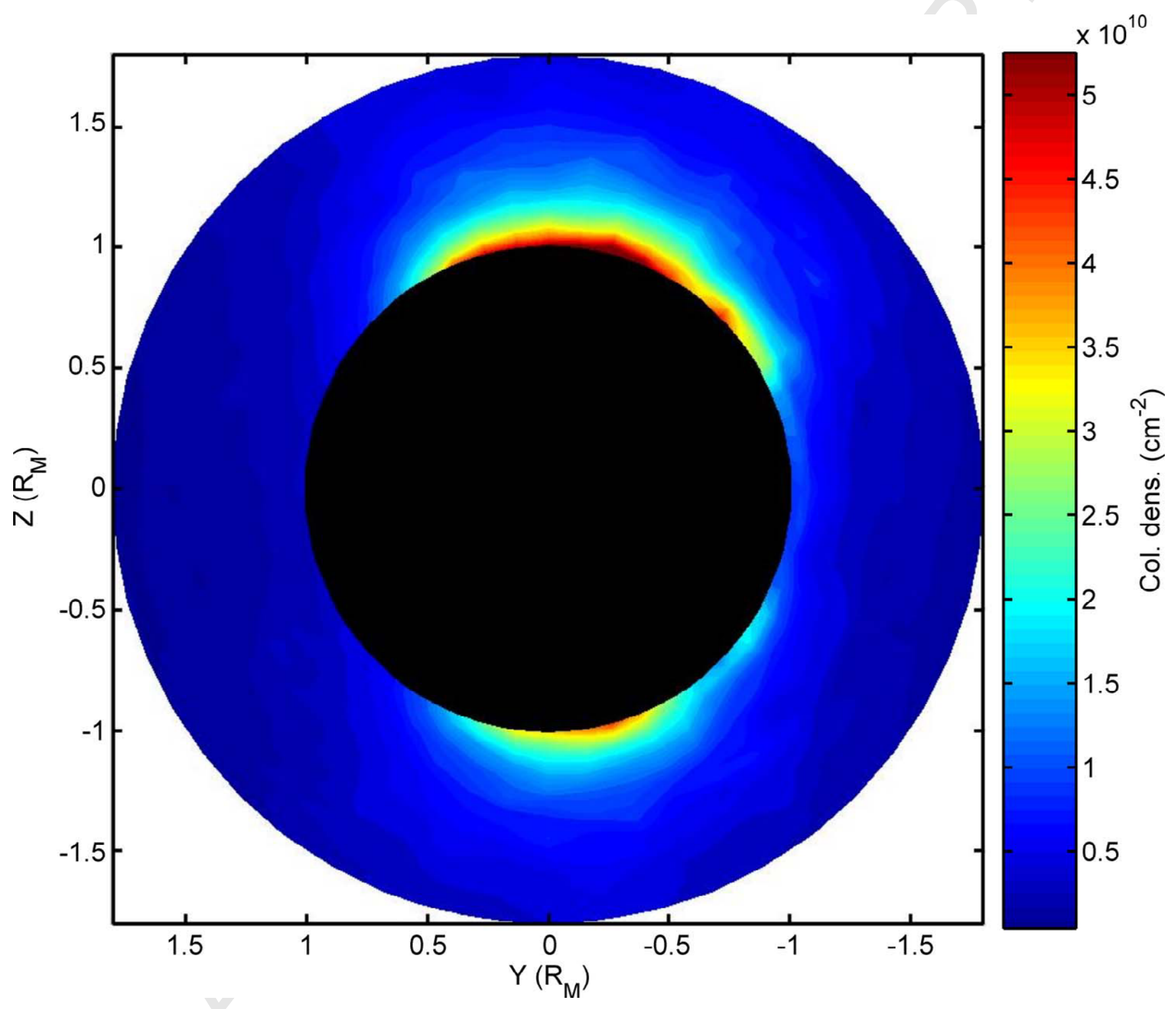




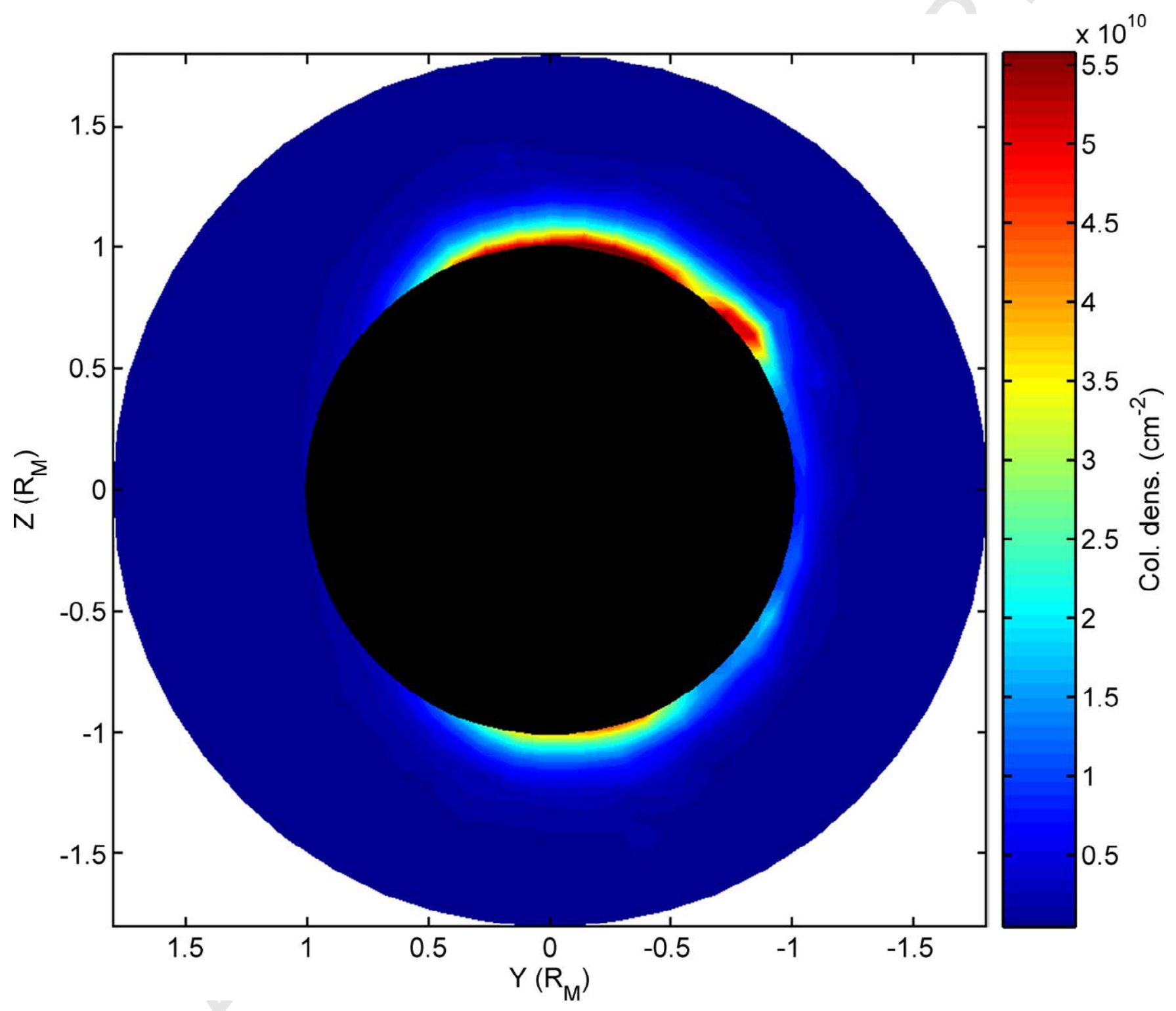




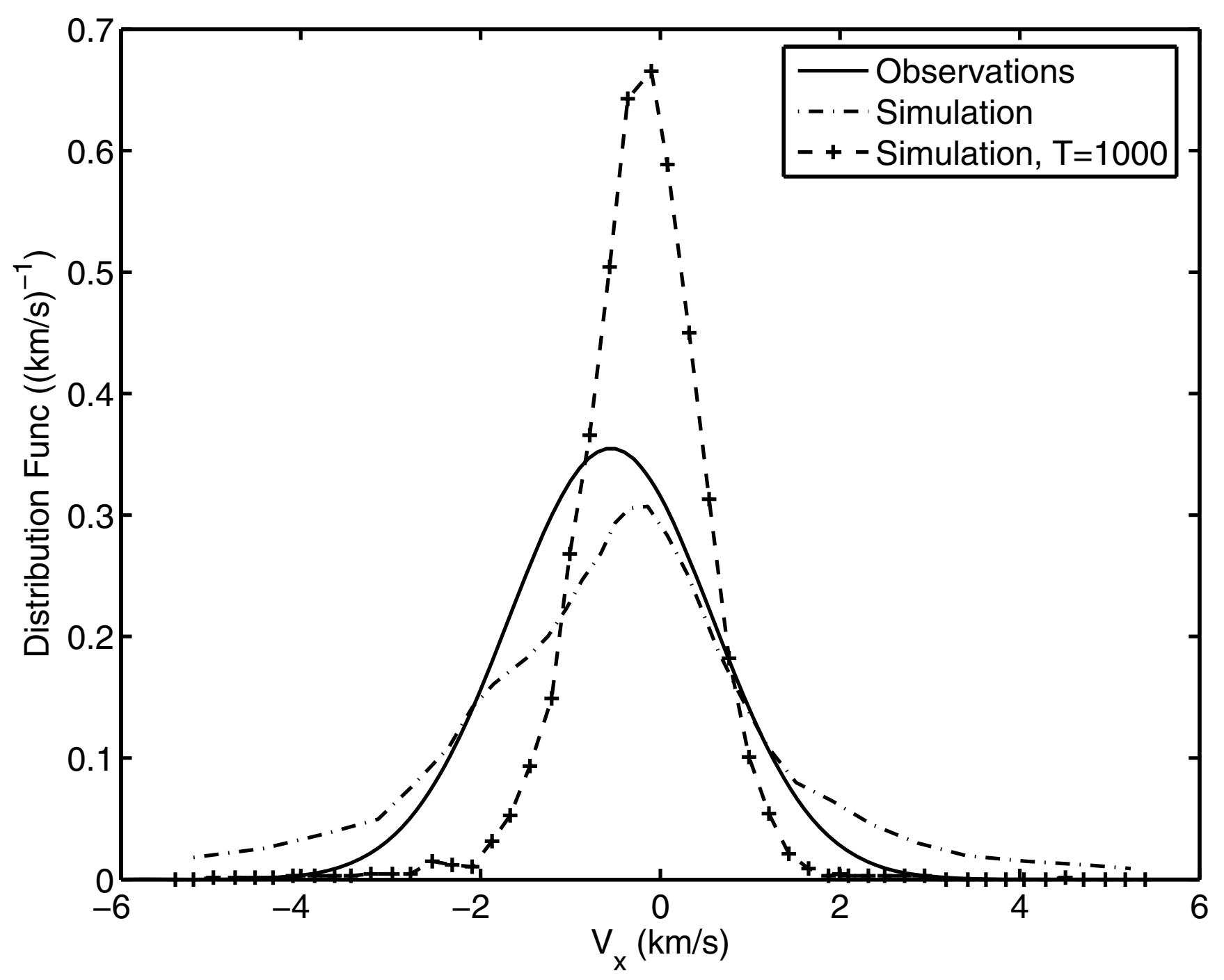




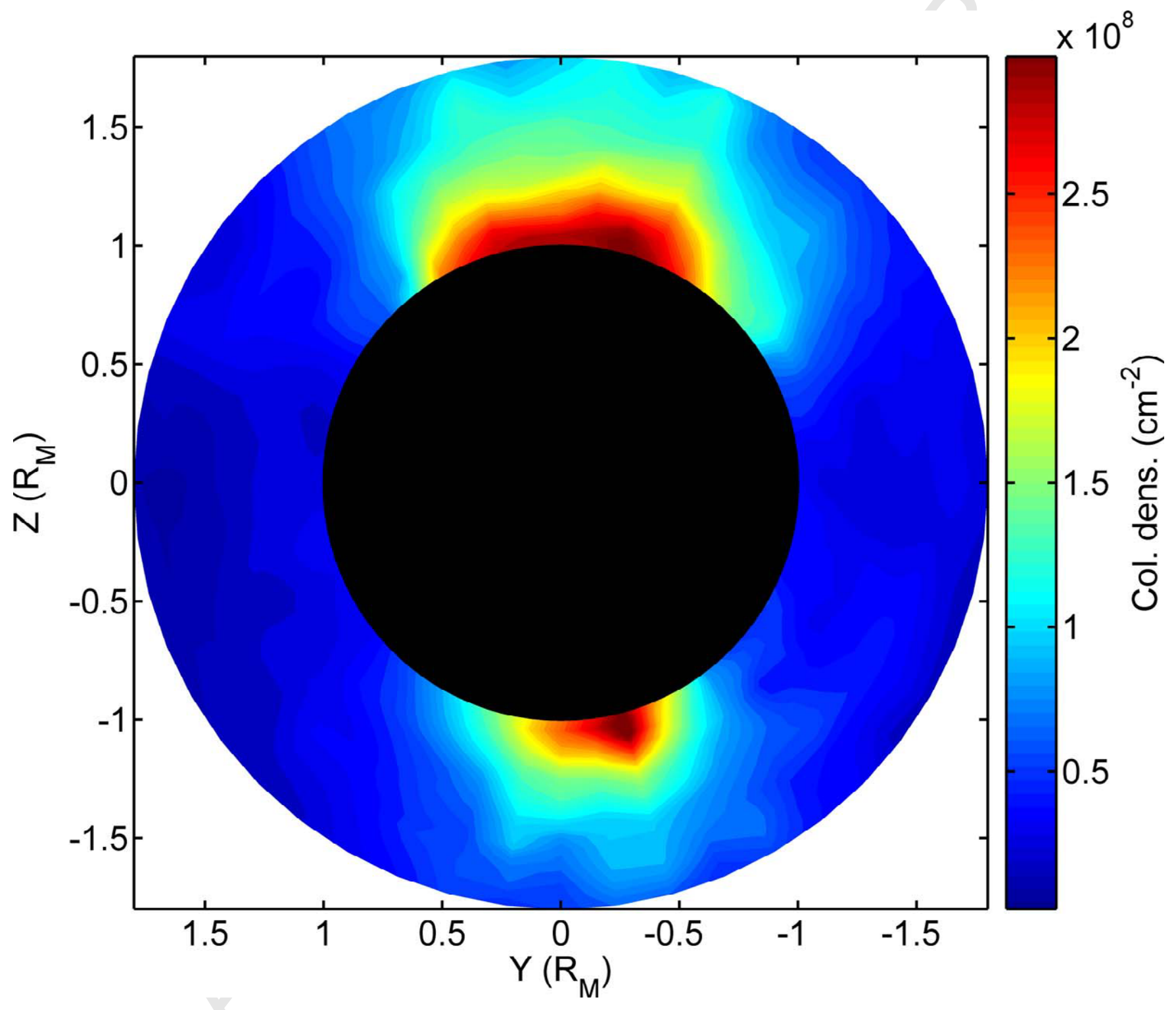




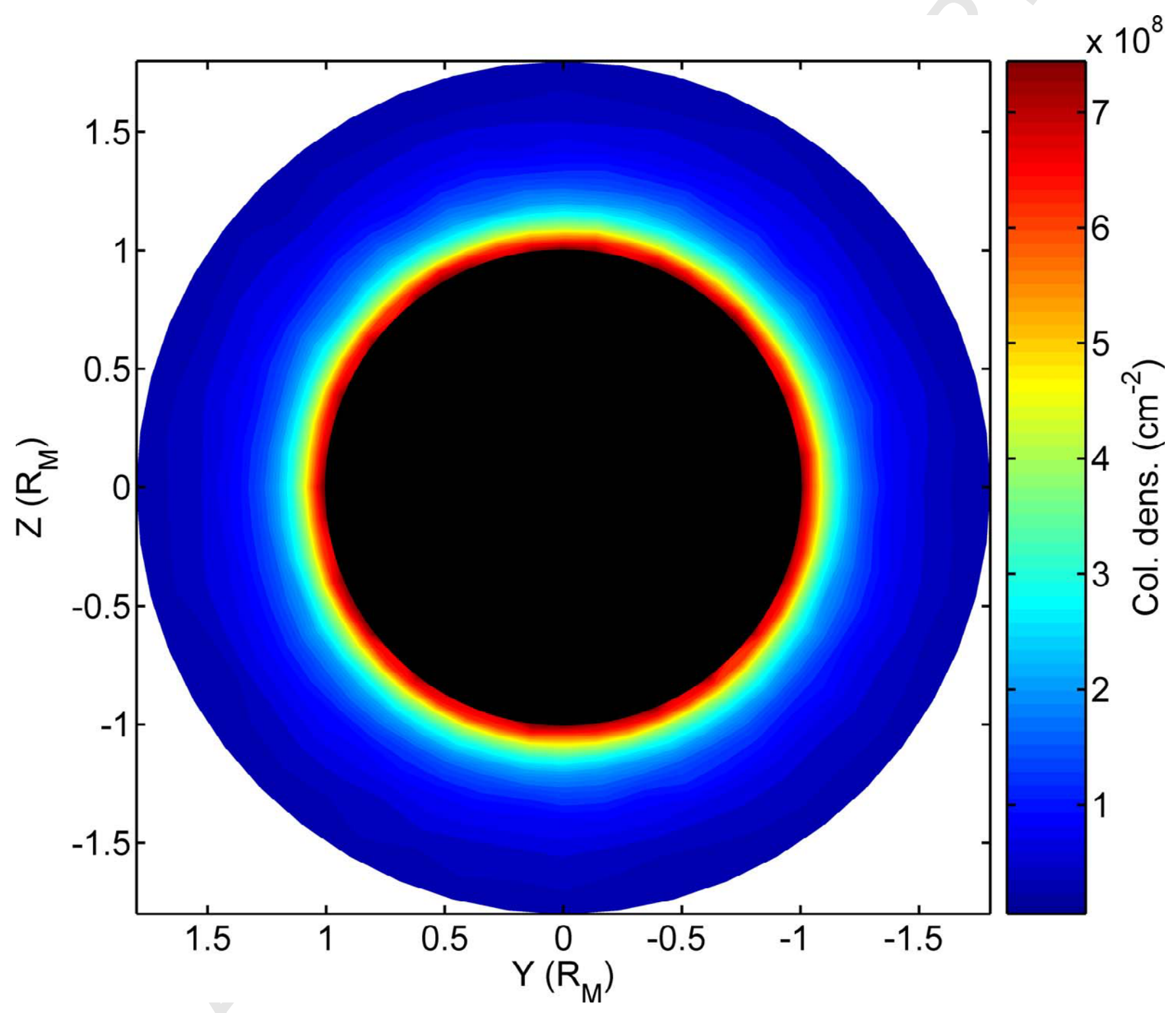




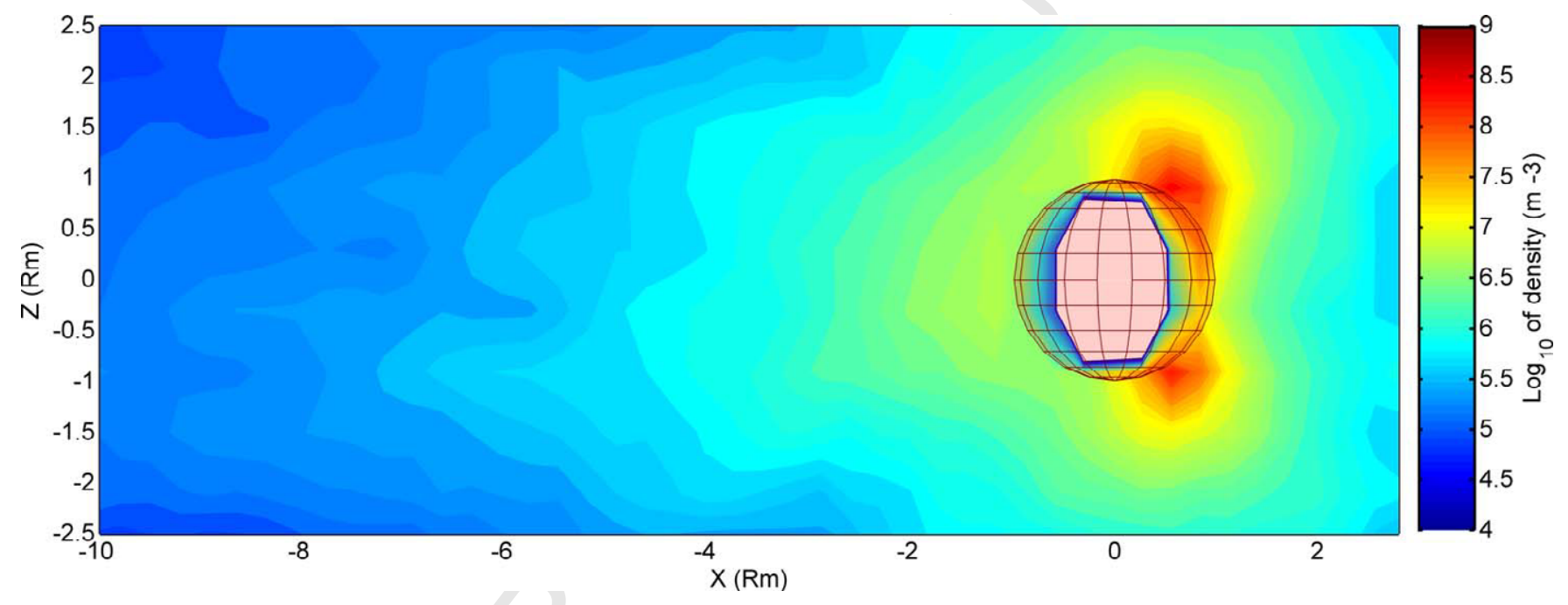

